\title{
Logros y desaciertos cuando se aprende a demostrar
}

\section{Successes and failures when learning to prove}

\author{
Luis Fernando Lara Quintero \\ Secretaría de Educación Distrital, Bogotá, Colombia \\ luisfernandolara26@yahoo.es \\ Carmen Samper \\ Universidad Pedagógica Nacional, Departamento de Matemáticas, Bogotá, Colombia \\ csamper@pedagogica.edu.co
}

RESUMEN • En este artículo presentamos algunos de los resultados obtenidos en un estudio de caso que permitió caracterizar los argumentos de un grupo de estudiantes (14-16 años) cuando, en el marco de la actividad demostrativa, formularon y justificaron una conjetura como respuesta a un problema geométrico. Particularmente, mostramos dos momentos en los que parece que los estudiantes entendieron qué es demostrar y cómo se construye una demostración, proceso que se vio afectado por dos conflictos epistémicos. El análisis de los argumentos de los estudiantes, a partir del modelo de Toulmin, nos permite establecer logros y desaciertos con respecto a la demostración que se evidenciaron.

PALABRAS CLAVE: actividad demostrativa; tipo de argumento; logros y desaciertos; conflicto epistémico.

ABSTRACT • In this article, we present some results obtained in a case study in which the arguments of a group of students, aged 14 to 16, were characterized, using Toulmin's model. The arguments were produced when, in a proving activity experience, they formulated a conjecture as the solution to a geometric problem and then justified it. Particularly, we show two moments in which there is evidence of the student's possible comprehension of what proving is and how proofs are constructed, process that was affected by two epistemic conflicts. The analysis of the arguments lets us establish successes and failures with respect to proof that were evidenced during the process.

KEYWORDS: proving activity; type of argument; success and failure; epistemic conflict. 


\section{INTRODUCCIÓN}

Con la intención de introducir a los estudiantes de grado noveno de educación básica a la argumentación matemática, se modificó la metodología usual de enseñanza incluyendo por primera vez tareas que requerían el uso de geometría dinámica. Esto permitió que los estudiantes realizaran actividad demostrativa (Perry, Samper, Camargo y Molina, 2013), al explorar una situación geométrica presentada por el profesor, formular una conjetura respecto al hecho geométrico, desconocido para ellos, que subyacía a la situación, y tratar de justificarla. Esta actividad fomentó la argumentación y promovió la participación de los estudiantes. A partir del análisis de los argumentos de los estudiantes durante el proceso de justificación, usando el modelo de Toulmin, fue posible identificar logros y desaciertos de ellos respecto a qué es demostrar y cómo se produce una demostración. Son los logros y desaciertos lo que queremos comunicar en el presente artículo, con el fin de ilustrar problemáticas que debe tener en cuenta un profesor cuyo interés es enseñar a demostrar. Con ello pretendemos contribuir en algo los resultados de investigaciones sobre dificultades que tiene los estudiantes para aprender a demostrar (Selden y Selden, 2011; Perry, Camargo, Samper y Rojas, 2006).

A continuación, describimos los antecedentes que suscitaron el problema que orientó la investigación; presentamos algunos referentes teóricos que sustentan el estudio y aspectos metodológicos del mismo. En seguida, damos a conocer el análisis hecho cuando los estudiantes comenzaron a justificar su conjetura. Terminamos con algunos comentarios y preguntas.

\section{CUESTIÓN DE LA INVESTIGACIÓN Y ANTECEDENTES DEL ESTUDIO}

El objetivo de la investigación que realizamos, de la cual surgen los datos que usamos en este artículo, era analizar el comportamiento argumental de los estudiantes cuando trabajaban en grupo dentro de un ambiente que propició la actividad demostrativa (Fonseca y Lara, 2013). Nuestro interés como profesores de secundaria era proponer tareas en el aula que favorecieran la argumentación entre estudiantes porque esta es un elemento esencial para entender lo que es una demostración y aprender a construirla. Nuestra hipótesis era que el uso de la geometría dinámica fomentaría la comunicación entre los estudiantes, logrando con ello evidenciar sus argumentos al interactuar sobre asuntos matemáticos.

Para lograr dicho objetivo fue necesario buscar investigaciones existentes que se hubiesen centrado en el estudio de la demostración en el aula escolar. Encontramos que algunos investigadores, como Pedemonte (2005) y Godino y Recio (2001), identifican problemáticas relacionadas con la enseñanza y el aprendizaje de la demostración. Hanna (1996; citada en Arzarello, Olivero, Paola y Robutti, 2007) señala que en las pocas escuelas donde se aborda la demostración, a pesar de creer que este es un aspecto central en matemáticas, prevalece el aprendizaje memorístico, lo cual carece de valor educativo y no tiene que ver con la actividad matemática correspondiente. Esto conlleva un bajo nivel en la comprensión y elaboración de demostraciones (Godino y Recio, 2001). Además, Jones (2000) menciona que los estudiantes no ven la necesidad de hacer demostraciones deductivas porque se ha privilegiado la verificación y se ha dejado a un lado la exploración y la explicación. Para enfrentar esta realidad y adoptando una perspectiva sociocultural del aprendizaje, el grupo de investigación Aprendizaje y Enseñanza de la Geometría $(\mathcal{A} \cdot \mathcal{G}$ ), de la Universidad Pedagógica Nacional de Colombia, ha propuesto estrategias metodológicas que favorecen la actividad demostrativa, tanto en el contexto universitario como en el escolar, y por ende el desarrollo de habilidades argumentativas. 


\section{REFERENTES TEÓRICOS}

Los constructos teóricos que respaldan lo que reportamos son: actividad demostrativa y modelo de Toulmin para la argumentación. Dichos constructos los tratamos a continuación.

\section{Actividad demostrativa}

El grupo de investigación $\mathscr{A} \cdot \mathcal{G}$ asume, en consonancia con De Villiers (1993), que la demostración, además de la validación, cumple funciones tales como ser medio de comunicación, explicación, sistematización y descubrimiento. Por esto, al admitir la necesidad de que los estudiantes realicen acciones propias de la comunidad matemática, y en la búsqueda de alternativas para que la demostración (en geometría) juegue un papel importante y significativo en el aprendizaje, el grupo $\mathcal{A} \cdot \mathcal{G}$ propone para el ámbito escolar y universitario lo que denominan actividad demostrativa (Camargo, Samper, y Perry, 2006). Un principio base del constructo actividad demostrativa es que la demostración en matemáticas tiene el propósito de proporcionar comprensión y conocimiento, además de cumplir las funciones mencionadas.

Teniendo en cuenta lo anterior, el grupo $\mathscr{A} \cdot \mathcal{G}$ concibe la actividad demostrativa como la realización de dos procesos, no necesariamente independientes (Perry et al., 2013). En el primer proceso, o de conjeturación, se establecen conjeturas a partir de las evidencias que provee la exploración de la situación con geometría dinámica, lo cual genera un alto grado de seguridad de su validez. Las acciones que hacen parte de este proceso son: detectar y verificar propiedades, formular y corroborar conjeturas. En el segundo proceso, o de justificación, se valida, si es posible, la conjetura dentro de un sistema teórico o con explicaciones empíricas, según el respectivo nivel escolar. Precisamente, el objetivo de nuestra investigación requería lograr que los estudiantes pasaran de formular argumentos empíricos, que usualmente hacían, a producir un discurso argumentativo de tipo deductivo con el cual validaran la conjetura formulada. Para ello, durante los dos meses que duró nuestra intervención en el aula, fue necesario conformar un sistema teórico al cual pudieran recurrir para sus argumentos. Las acciones que conforman el proceso de justificación son: seleccionar elementos teóricos o empíricos, construir argumentos y formular la justificación. Aceptamos elementos empíricos dado que reconocíamos que el cambio de argumentos informales a justificaciones formales difícilmente se lograría en tan corto tiempo.

\section{Modelo de Toulmin}

El modelo de argumentación propuesto por Toulmin es una herramienta útil para analizar los argumentos que los estudiantes producen durante el desarrollo de la actividad demostrativa (Lavy, 2004; Pedemonte, 2007; Boero, Douek, Morselli y Pedemonte, 2010; Perry et al., 2013). Asumimos como argumento un enunciado oral o escrito, utilizado para convencerse o para convencer a otros. De acuerdo con el modelo, un argumento está estructurado a partir de tres elementos principales y tres secundarios. Los elementos principales, que conforman lo que se conoce como el modelo básico de Toulmin (Rodríguez y Gutiérrez, 2012), son: datos o información, conclusión expresada como una afirmación que se cree que es consecuencia de los datos y garantía, que es una regla (axioma, definición o teorema) que relaciona los datos con la conclusión. La manera como los tres elementos principales, que no necesariamente están explícitos en un argumento, se estructuran y relacionan definen tres tipos de argumentos (Perry et al., 2013): deductivo, abductivo ${ }^{1}$ o inductivo. Para cada uno de ellos, en los esque-

1. En los argumentos abductivos, la conclusión se acepta como un hecho y a partir de garantías conocidas, ya sean reglas hipotéticas o teóricas que podrían justificar a la conclusión, se buscan datos adecuados que son provisionales. 
mas que presentamos en las figuras 1,2 y 3 , el producto que se obtiene es diferente y lo indicamos con un recuadro punteado.

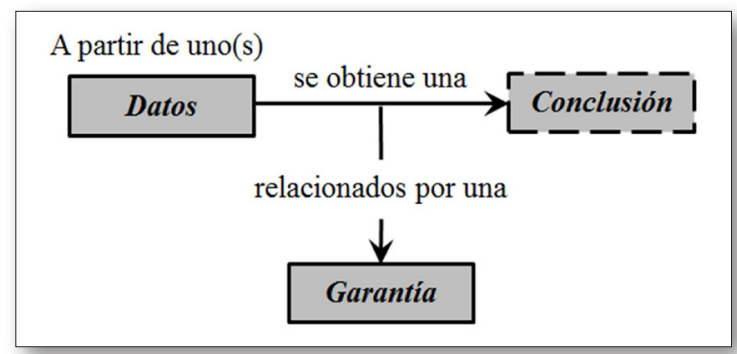

Fig. 1. Esquema de un argumento deductivo.

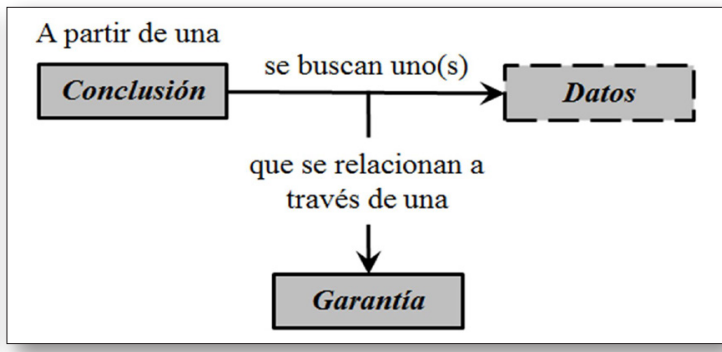

Fig. 2. Esquema de un argumento abductivo.

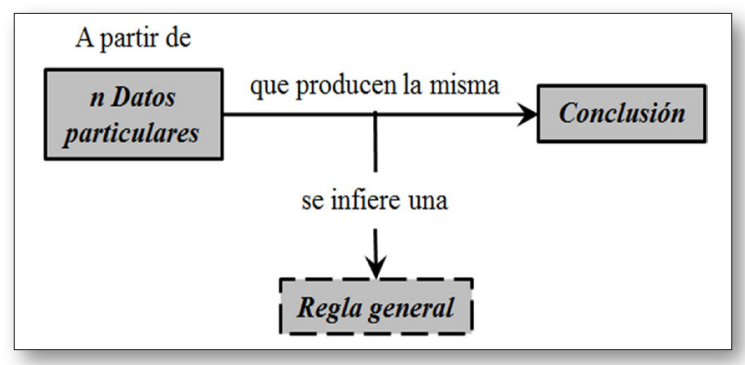

Fig. 3. Esquema de un argumento inductivo.

Con el fin de complementar el análisis de un argumento, el modelo incluye tres elementos secundarios: un cualificador modal o indicador de fuerza del argumento (quizá, probablemente, por lo que parece, etc.), una refutación o excepción de la conclusión o de la garantía (objeciones, contraejemplos, etc.) y un respaldo o apoyo a la garantía que es necesario si esta no es aceptada de inmediato (Rodríguez y Gutiérrez, 2012; Toulmin, 2003). Entonces, el modelo completo de Toulmin consta de seis elementos relacionados, como mostramos en el diagrama de la figura 4.

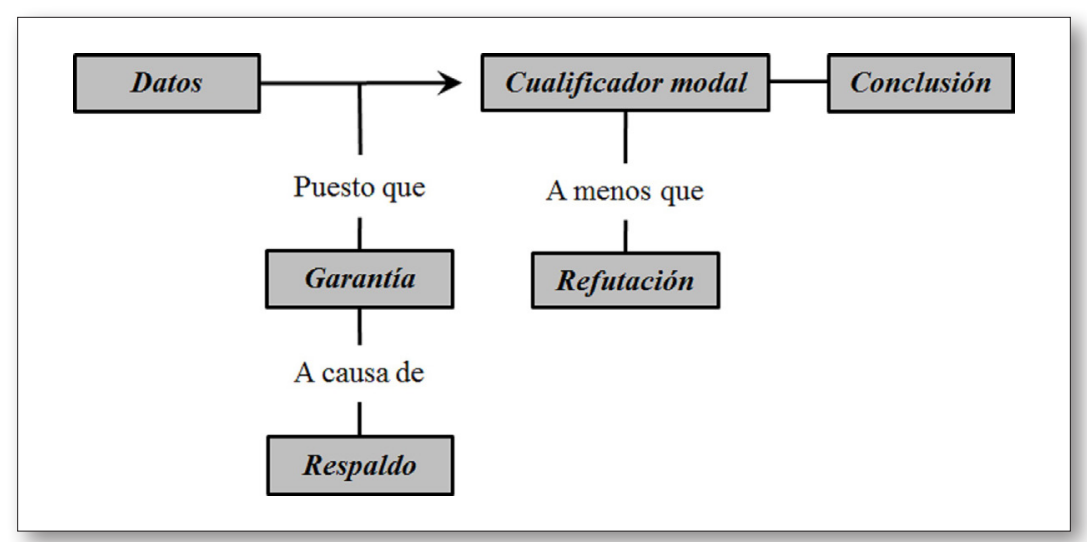

Fig. 4. Diagrama del modelo completo de Toulmin. 


\section{MARCO METODOLÓGICO}

En este apartado, presentamos brevemente el proceso que permitió desarrollar dicho estudio. Específicamente, describimos el tipo de investigación, el contexto en el que se llevó a cabo y las fases del estudio.

\section{Tipo de investigación}

Adoptamos una metodología cualitativa centrada en la corriente descriptiva-interpretativa que corresponde a un estudio de caso. De acuerdo con Cohen y Manion (1990), dicho estudio de caso fue, en primer lugar, de tipo no participante porque el profesor evitó involucrarse en las discusiones de los estudiantes durante los dos procesos de la actividad demostrativa. En segundo lugar, fue estructurado porque usamos la aproximación metodológica del grupo $\mathscr{A} \cdot \mathcal{G}$ con el fin de generar un entorno favorable para aprender a demostrar. Esta aproximación se describe más adelante.

\section{Contexto de la investigación}

Diseñamos e implementamos una secuencia didáctica en un colegio público de Bogotá (Colombia) con estudiantes de grado noveno de educación básica secundaria (14-16 años). Esta duró aproximadamente dos meses del segundo semestre de 2011, tiempo en el que desarrollamos 13 sesiones de clase de hora y media. El estudio de caso se hizo sobre la actividad de un grupo de dos estudiantes del curso, Diana y Cristian, cuando resolvían dos problemas al finalizar los dos meses. Elegimos este grupo porque trabajaron juntos durante gran parte del desarrollo de la secuencia didáctica y mantuvieron un intercambio comunicativo bueno. En términos generales, Diana se destacaba en matemáticas y Cristian tenía un rendimiento académico promedio. El profesor solo intervino en las discusiones de los estudiantes cuando estas se estancaban porque no podían llegar a un acuerdo, o para solicitar alguna aclaración de lo que se estaba diciendo.

\section{Fases de la investigación}

El desarrollo del estudio estuvo conformado por dos fases. En la primera fase, diseñamos e implementamos la secuencia didáctica cuyo propósito era introducir los elementos teóricos para que los estudiantes, en un momento determinado, pudieran formular y demostrar una conjetura. Usamos la aproximación metodológica en el aula propuesta por el grupo $\mathcal{A} \cdot \mathcal{G}$ porque han mostrado que se genera un ambiente propicio para aprender a demostrar (Perry et al., 2013). La aproximación metodológica se caracteriza por tres elementos. El primero es las tareas que favorecen la visualización, la exploración y la formulación de conjeturas que dan pie a la introducción de elementos teóricos. La organización, con el apoyo del profesor, de estos como definiciones o como hechos geométricos es lo que llamamos un sistema teórico local. El segundo elemento de la aproximación metodológica es la interacción social en el aula entre profesor y estudiantes, y entre estudiantes, que permite la comunicación y discusión de ideas que se validan o rechazan a través de la argumentación. Y el tercero es el uso de geometría dinámica (Cabri), que favorece la construcción y exploración de propiedades geométricas y permite que los estudiantes produzcan conjeturas. Dicha secuencia didáctica se desarrolló en tres etapas: ${ }^{2}$ en la primera (seis sesiones) se comenzó a conformar el sistema teórico local cuyo núcleo eran los criterios de con-

2. En Fonseca, Lara y Samper (2012) se describe con detalle algunas de las tareas que diseñamos y aplicamos durante la secuencia didáctica. 
gruencia de triángulos; en la segunda (cinco sesiones), por medio de tareas en las que se usó Cabri, se amplió el sistema teórico, incluyendo teoremas como el que afirma la congruencia de ángulos opuestos por el vértice y definiciones como la de bisectriz de ángulo y distancia de un punto a una recta. En el sistema teórico local así conformado se encontraban los elementos teóricos necesarios para resolver el problema que llevó a la formulación de una conjetura y a su respectiva justificación, actividad que consiste en la tercera etapa (dos sesiones).

En la segunda fase, grabamos en audio y video cada una de las sesiones del desarrollo de la secuencia didáctica, recogimos las producciones escritas de los estudiantes y transcribimos la interacción de los estudiantes durante las dos últimas sesiones; todas las sesiones estuvieron a cargo del profesor titular de la institución, quien es uno de los autores del presente artículo. De acuerdo con el modelo básico de Toulmin y con la descripción que el grupo $\mathscr{E} \cdot \mathcal{G}$ hace de los tipos de argumentos, identificamos y clasificamos los argumentos de los estudiantes en deductivos, abductivos o inductivos. Además, establecimos dos subcategorías en relación con la manifestación explícita o no de alguno de los tres elementos principales que conforman un argumento (datos, conclusión y garantía). De tal manera que los llamamos argumentos completos cuando están explícitos los tres elementos del argumento, y argumentos incompletos cuando falta alguno de ellos. En cuanto a los elementos secundarios de un argumento propuestos por Toulmin, evidenciamos que en ningún momento los estudiantes mencionan algún respaldo para la garantía. Generalmente sentían seguridad respecto a su argumento, pues no emitieron expresiones que indicaran la plausibilidad de estos (cualificador modal). En contados momentos los estudiantes se refirieron explícitamente a la fuerza del argumento. En ese caso, si hubo refutación lo indicaremos.

Para hacer el análisis dividimos las transcripciones en episodios, determinados a partir de la acción principal realizada; ilustramos las acciones de los estudiantes con gráficas capturadas de los videos y escaneamos sus producciones escritas; hicimos anotaciones en paréntesis cuadrados para complementar las intervenciones que involucraron acciones no verbales, y eliminamos aquellas intervenciones que se referían a cuestiones ajenas a la solución del problema. Los extractos de la interacción de los estudiantes, con su respectivo análisis, que se escogieron ilustran precisamente lo que consideramos como evidencia de los logros y desaciertos en el proceso de producción de una justificación.

\section{ANÁLISIS DE DATOS Y RESULTADOS}

En la primera sesión de clase previa a la actividad que vamos a analizar en este artículo, los estudiantes resolvieron el problema 1: Don Gustavo es un campesino que desea cultivar arroz en su finca. Para ello, tiene que inundar el potrero en que sembrará las matas de arroz, sacando agua de una canal. Hay una llave de agua en el punto P lejos de la canal. Por tanto, debe construir una tubería desde el punto P a un punto de la canal para llenarla de agua. Si don Gustavo quiere que la construcción sea lo más económica posible, entonces ¿en dónde debe localizar un punto $Q$ sobre la canal para que pueda cumplir con su intención? Con este problema los estudiantes descubrieron que la mínima distancia corresponde a la longitud del segmento perpendicular a la canal con extremos el punto $P$ y un punto de esta, hecho que se incluyó en el sistema teórico como la definición de distancia de un punto a una recta. En la segunda sesión previa, formularon una conjetura basada en su solución del problema 2: Uno de los terrenos en la finca de don Gustavo tiene forma de cuña, bordeado por dos canales. Él quiere sembrar matas de arroz de tal forma que la distancia de cada mata a cada canal sea la misma (figura 5). ¿Cuántas de estas puede sembrar? ¿Cómo se puede describir el sitio en donde don Gustavo debe colocar las matas? 


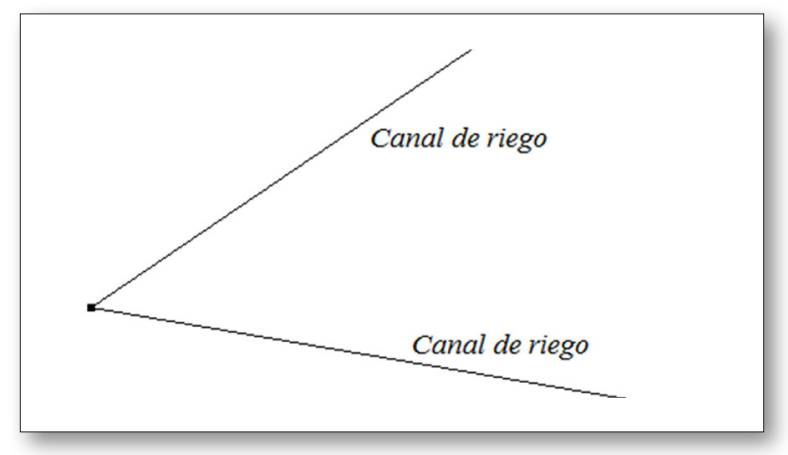

Fig. 5. Representación de la situación.

Los momentos clave del proceso de conjeturación al resolver el problema 2 se mencionan en la tabla 1.

Tabla 1.

Momentos clave del proceso de conjeturación del problema 2

\begin{tabular}{|c|c|c|}
\hline Momento clave & Protagonista & Acciones \\
\hline 1. Anticipan una respuesta. & Cristian & $\begin{array}{l}\text { Menciona que las matas deben estar en el centro de la } \\
\text { región angular. }\end{array}$ \\
\hline $\begin{array}{l}\text { 2. Representan la situación con geome- } \\
\text { tría dinámica y buscan la posición de } \\
\text { una mata. }\end{array}$ & Cristian & $\begin{array}{l}\text { Representa la mata con un punto } K \text {; construye un seg- } \\
\text { mento con extremos en los lados del ángulo; arrastra el } \\
\text { punto } K \text { para que sea el punto medio del segmento. }\end{array}$ \\
\hline $\begin{array}{l}\text { 3. Modifican la forma de encontrar la } \\
\text { posición de una mata. }\end{array}$ & Diana & $\begin{array}{l}\text { Rechaza la propuesta de Cristian, recuerda lo que signifi- } \\
\text { ca distancia de un punto a una recta y propone construir } \\
\text { segmentos perpendiculares a los lados del ángulo. }\end{array}$ \\
\hline 4. Encuentran la posición de otra mata. & Cristian & $\begin{array}{l}\text { Construye segmentos perpendiculares a los lados del } \\
\text { ángulo con extremos en } K \text { y en un punto del lado } \\
\text { correspondiente; toma la longitud de los segmentos y } \\
\text { arrastra hasta que son iguales. }\end{array}$ \\
\hline $\begin{array}{l}\text { 5. Concluyen que hay infinitas posicio- } \\
\text { nes para las matas. }\end{array}$ & Cristian & $\begin{array}{l}\text { Señala en la representación en la pantalla de la calcula- } \\
\text { dora otras posiciones del punto con un lápiz. }\end{array}$ \\
\hline $\begin{array}{l}\text { 6. Determinan cómo describir el lugar } \\
\text { geométrico de las matas. }\end{array}$ & Diana & $\begin{array}{l}\text { Identifica que los puntos son colineales y pertenecen a la } \\
\text { bisectriz del ángulo. }\end{array}$ \\
\hline 7. Establecen la conjetura. & Diana & $\begin{array}{l}\text { Dicta: «Si el ángulo } C R I \text { con un punto dentro de él }[K] \\
\text { que llevaba la misma distancia de rayo a rayo entonces lo } \\
\text { que descubrimos que por medio de la bisectriz podía- } \\
\text { mos saber dónde colocar los puntos». }\end{array}$ \\
\hline
\end{tabular}

Para comenzar la sesión en la que van a justificar su conjetura, el profesor le solicitó a Cristian hacer un recuento del proceso que siguieron para resolver el problema, indicando cuál era su conjetura. Luego, conjuntamente, profesor y estudiantes establecieron la siguiente afirmación condicional que expresa la misma idea de la conjetura pero de una manera más formal: «Si la distancia de un punto a cada lado de un ángulo es igual, entonces el punto está sobre la bisectriz del ángulo». En seguida, los estudiantes comienzan a construir la justificación de dicha afirmación; consignan, una vez llegan a un acuerdo, cada paso de esta en el esquema que habían utilizado durante el desarrollo de la secuencia didáctica para organizar y comunicar la demostración. Este esquema, denominado por Samper, Molina y Echeverry (2011) como esquema-deducción, está conformado por tres columnas tituladas "Qué sé», 
"Qué uso» y "Qué concluyo» (tabla 2), en las cuales se registraban, respectivamente, el dato, la garantía proveniente del sistema teórico local conformado y la conclusión de cada argumento, paso de la justificación. A los estudiantes se les permitió usar una hoja en la que habían consignado las definiciones y los hechos geométricos establecidos durante los dos meses, si lo requerían.

El análisis que aparece enseguida corresponde al segundo proceso de la actividad demostrativa, en el cual logramos identificar dos actuaciones de los estudiantes que son evidencia de comprensión sobre lo que significa demostrar en matemáticas (logros). La primera es la formulación de tres argumentos completos, relacionados con la información suministrada en la afirmación que hay que demostrar pero no encadenados, por lo que no son pasos de la justificación esperada. Los llamamos argumentos situación (AS). Esto sucede en los episodios 1 y 2. La segunda actuación es cuando Diana propone escoger uno de los tres argumentos completos para usarlo como el primer paso de la justificación, y, juntos, los alumnos construyen los demás pasos, incorporando en algún momento los otros dos AS. Cada paso de la demostración lo llamamos argumento paso (AP). Esto sucede durante los episodios 3 y 4.

En el análisis identificamos dos conflictos epistémicos que fueron obstáculo para el desarrollo de la justificación (desaciertos); por ello, se presentaron avances y retrocesos en el proceso de construcción de la justificación, haciendo que este durara alrededor de tres horas. Sin embargo, los alumnos culminaron con éxito la tarea.

\section{Episodio 1: Construyen el primer AS}

Inicialmente, los estudiantes mantienen una conversación en la que identifican qué saben (antecedente de la condicional) y qué deben justificar (consecuente de la condicional), sin recurrir a la construcción hecha en Cabri ni realizar una representación con lápiz y papel. Por sugerencia del profesor, Cristian representa la situación con lápiz y papel (figura 6). En seguida, Diana menciona que «La distancia del punto $K \ldots$ ¿es igual a $C, Y$ ? No, no se puede. ¿Entonces?». Mientras que Cristian señala: «Pues no sabemos es cómo redactarla (equidistancia del punto $K$ a los lados del $\angle C Y A$ )». En este momento identificamos que tanto Cristian como Diana no saben cómo aplicar la definición de distancia de un punto a una recta a la situación específica del problema (desacierto). Esto se evidencia porque ninguno de los dos estudiantes se percata de la necesidad de trazar segmentos perpendiculares a los lados del ángulo y nombrarlos para lograr poner en términos de estos la equidistancia.

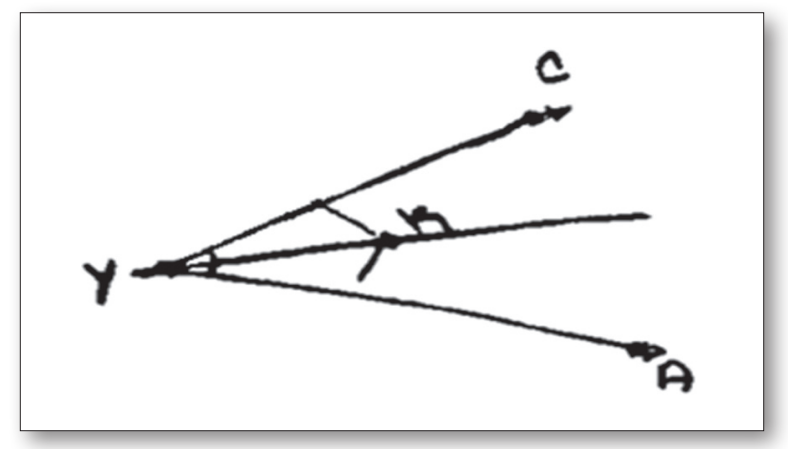

Fig. 6. Bosquejo de la situación.

Debido a la coyuntura en que se encuentran, los estudiantes reconocen que su representación de la situación es deficiente. Diana cree que debe asignarle una medida específica a los segmentos con extremo en $K$, idea que le comunica a Cristian: «¿Será que no se le puede colocar una medida a eso?... podríamos colocar que la distancia del punto $K$ es 5 , digamos $5 \mathrm{~cm}$ al segmento $C$, $Y$ ». Como Cristian 
no entiende inmediatamente a qué se está refiriendo, ella explica: «Ah, esos segmentos pero no están nombrados». Por ello, Cristian realiza algunos cambios en la representación que acompaña el enunciado del problema, sobre la cual Diana había hecho algunos trazos. Nombra con la letra $I$ al punto que tiene igual distancia a los lados del $\angle C Y A$ y con las letras $K$ y $J$ a los puntos que corresponden a los extremos de los dos segmentos trazados por Diana (figura 7). Cristian objeta la sugerencia de Diana de asignarle a los segmentos una medida específica. Aquí se enfrentan con otra problemática para aprender a demostrar: ser capaces de generalizar los ejemplos particulares que proponen los estudiantes para entender la situación o que surgen del uso de la geometría dinámica (desacierto).

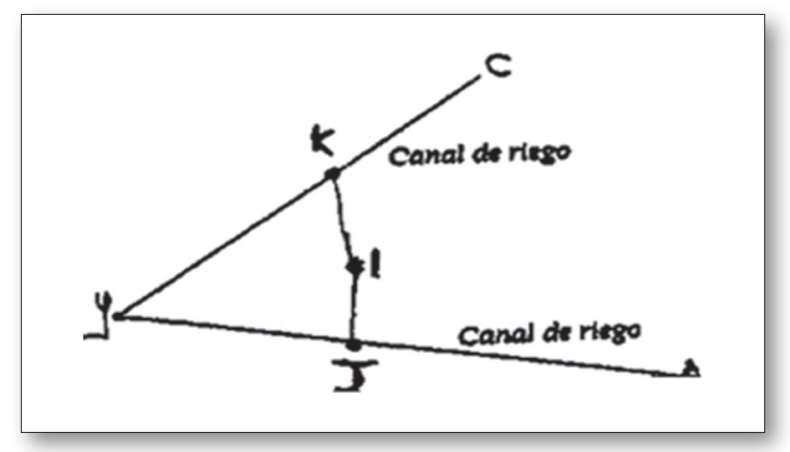

Fig. 7. Bosquejo con puntos nombrados.

196. ${ }^{3} \quad$ Cristian: $\quad$ El segmento $I, K$ y el segmento $I$, $J$ son congruentes. Sabemos eso. ¿̨Por qué? Porque miden lo mismo.

197. Diana: Entonces utilizaríamos la definición de segmentos congruentes. Si no es eso, mejor dicho.

[...]

258. Diana: [Lee la afirmación que se va a demostrar]. Si la distancia de un punto a cada lado de un ángulo es igual, entonces el punto está sobre la bisectriz del ángulo. Pero como todavía no podemos utilizar esa parte del entonces [señala el consecuente de la afirmación], porque eso es lo que debemos concluir, al final.

259. Cristian: Entonces, debemos saber esto $[\overline{K I}$ tiene la misma medida que $\overline{I J}$. $]$. Solo que lo debemos saber escribir [realiza una marca indicando el inicio y el final del antecedente de la afirmación: Si la distancia de un punto a cada lado de un ángulo es igual].

Cristian y Diana formulan conjuntamente un argumento deductivo completo. En [196] Cristian plantea como conclusión la congruencia entre el $\overline{I K}$ y el $\overline{I J}$, y como datos que dichos segmentos tienen la misma longitud. La correspondiente garantía la provee Diana en [197]. Ella muestra seguridad (cualificador modal) respecto al argumento construido conjuntamente. Mientras que Diana afirma en [258] que no se puede usar la conclusión de la afirmación como dato, Cristian dice en [259] que se debe partir del antecedente. Esto es evidencia de que ellos reconocen cuál es el papel tanto del antecedente como del consecuente de la afirmación en la construcción de su demostración (logro).

\section{Episodio 2: Formulan el segundo y tercer AS}

Terminado el primer argumento, Cristian propone involucrar ángulos. Comprueba en la calculadora la perpendicularidad entre los segmentos con extremo el punto $I$ y los lados del $\angle C Y A$, acto que

3. Los números indican el orden de intervenciones de las personas que participaron durante el segundo proceso de la actividad demostrativa: estudiantes y profesor. 
rechaza Diana al señalar que eso no es necesario porque en la construcción se empleó la herramienta recta perpendicular. La problemática que subyace a la propuesta de Cristian es la falta de comprensión de que demostrar una proposición condicional significa asumir como verdaderos los datos incluidos en el antecedente sin importar cómo es la representación de la situación (desacierto). En este caso, la perpendicularidad de los segmentos era un dato dado implícitamente. Diana traza nuevamente en la representación en papel los segmentos de forma que se vea que se cumple esa propiedad (figura 8).

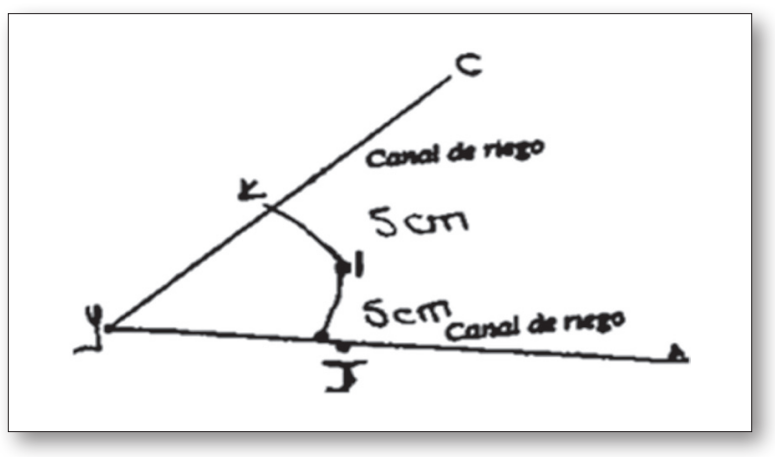

Fig. 8. Bosquejo con segmentos perceptualmente perpendiculares a los lados del ángulo.

Luego, tanto Diana como Cristian formulan argumentos distintos en torno a la perpendicularidad. En las siguientes intervenciones de Diana se puede apreciar un argumento:

¿Así [escribe en la columna Qué sé del segundo argumento: $\overline{I K} \perp \overline{Y C}$ ]? Entonces, ¿qué usamos? Pues la definición de rectas perpendiculares... [298]

Espere [...] [escribe en la columna Qué uso: definición de rectas perpendiculares]. Entonces lo que concluimos es que... iAy, acá no está [revisa en el listado]! [302]

No. Espere. Espere. Y, ¿qué concluimos? Que los ángulos $C, K, I$ es congruente con $Y, K, I$ [ $\angle C K I \cong \angle Y K I]$ ¿Sí me entiende? [345]

En este caso, Diana propone un argumento deductivo incompleto porque, a partir de la perpendicularidad entre el $\overline{I K}$ y el $\overline{Y C}$ (datos) y el uso de la definición de rectas perpendiculares (garantía), no puede deducir inmediatamente que $\angle C K I \cong \angle Y K I$, conclusión que ella propone. El hecho de usar la definición de rectas perpendiculares, sin haberla encontrado en su listado, es muestra de la seguridad (cualificador modal) que siente Diana respecto a su argumento. De manera rigurosa, a este proceso deductivo le hizo falta un argumento si hubiera usado el hecho geométrico de ángulos rectos, o dos argumentos si hubiera empleado la definición de ángulos rectos y la definición de ángulos congruentes (ver recuadros punteados de las tablas 2 y 3 , respectivamente).

Tabla 2.

Proceso deductivo de Diana con un argumento adicional

\begin{tabular}{|c|c|c|}
\hline Quésé & Qué uso & Qué concluyo \\
\hline$\overline{I K} \perp \overline{Y C}$ & $\begin{array}{c}\text { Definición rectas } \\
\text { perpendiculares }\end{array}$ & $\angle C K I$ y $\angle Y K I$ son rectos \\
\hline & & $\angle C K I \cong \angle Y K I$ \\
\hline$\quad \angle C K I$ y $\angle Y K I$ son rectos & $\begin{array}{c}\text { Hecho geométrico ángulos } \\
\text { rectos }\end{array}$ & \\
\hline
\end{tabular}


Tabla 3 .

Proceso deductivo de Diana con dos argumentos adicionales

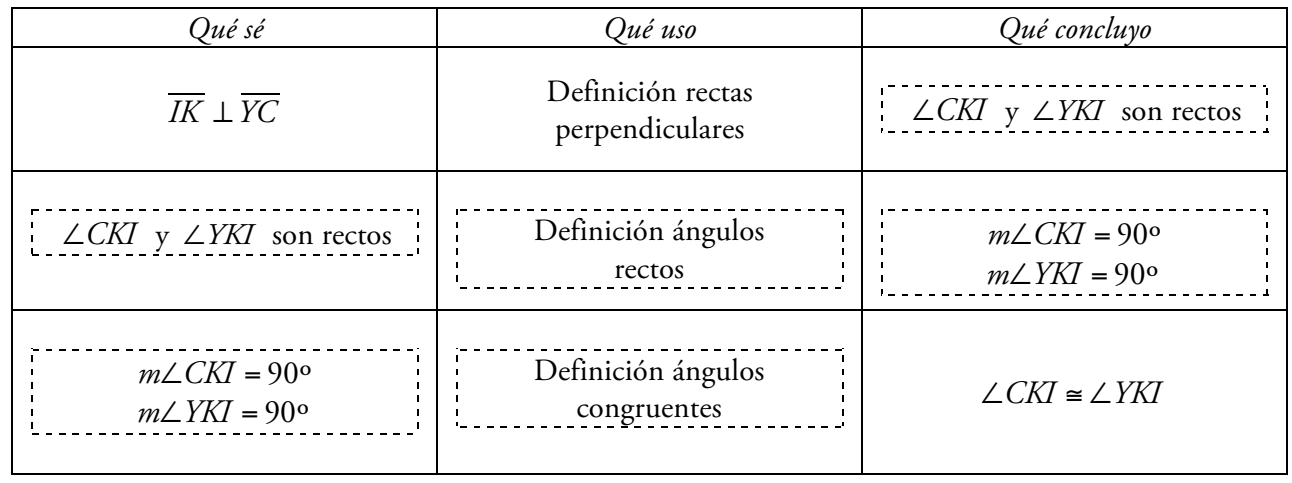

Por su lado, Cristian propone dos argumentos. Como primer argumento, él dice:

Pero esto [señala la afirmación $\overline{I K} \perp \overline{Y C}$ ] toca escribirlo acá [en la columna Qué concluyo y no en la columna Qué sé]. [299]

Póngale cuidado. Se supone que acá se forman dos ángulos rectos, ¿̇cierto [señala el $\angle Y K I$ y el $\angle C K I$ ]? [316]

Entonces, eso es lo que hay que escribir [en la columna Qué sé del segundo argumento]. [320]

El anterior argumento es de tipo abductivo completo, pues aunque no lo dice explícitamente, con la misma garantía empleada por Diana en su argumento anterior (definición de rectas perpendiculares), y con la conclusión que indica en [299] $(\overline{I K} \perp \overline{Y C})$, plantea como datos que se tienen dos ángulos rectos $(\angle Y K I$ y $\angle C K I)$.

Con relación al segundo argumento, él afirma:

Póngale cuidado. Sabemos que [el ángulo] $I, K$, y $C$ [y el ángulo $I, K$, e $Y$ ] son ángulos rectos. [334]

Entonces, utilizamos esa definición [no específica a qué elemento teórico realmente se refiere] y concluimos que [...] [el ángulo] $I, K, C$ y [el ángulo] $I, K, Y$ son congruentes [ $\angle I K C \cong \angle I K Y$ ]. [338]

Este segundo argumento es deductivo incompleto porque, con los mismos datos de su argumento abductivo anterior [334] y la garantía que no especifica en [338], no puede concluir inmediatamente la congruencia de los ángulos, al igual que Diana. Si la garantía a la que alude es la definición de ángulos rectos, entonces le hace falta seguir el argumento como se indica en la segunda y tercera filas de la tabla 3; si la garantía es la definición de ángulos congruentes, le falta concluir previamente que los ángulos miden $90^{\circ}$ (tercera fila de la tabla 3 ).

Consideramos que los argumentos de Diana y Cristian son incorrectos porque en el proceso deductivo constituido por una cadena de argumentos los estudiantes omiten uno o varios de estos, tal y como se muestra en los anteriores esquemas-deducción (tablas 2 y 3 ).

Posteriormente, Cristian cambia los datos de su primer argumento por ángulos con medida igual a $90^{\circ}$, con el fin de consignar en el esquema su segundo argumento:

El ángulo $I, K$, y $C$ sabemos que mide $90^{\circ}$ [escribe en la columna Qué sé del segundo argumento: $\left.\angle I K C \quad 90^{\circ}\right]$. ¿Cierto? [358]

Y el ángulo $I$, $K$, e $Y$ también mide 90º [continúa escribiendo en la columna Qué sé: $\angle I K C \quad 90^{\circ}$ ]. [360]

Puedo sacar esto [señala la columna Qué uso del segundo argumento: definición de rectas perpendiculares]. [362] 
Entonces, podemos concluir que el segmento $I, K$ [escribe en la columna Qué concluyo del segundo argumento: $\overline{I K} \perp$ ] es [368]

[...] perpendicular con $Y, C[\ldots]$. Eso es lo que ya [...] concluimos. Y se puede sacar la misma conclusión con el de abajo $[\overline{I J} \perp \overline{Y A}]$. [384]

El argumento deductivo completo de Cristian es incorrecto de acuerdo con el sistema teórico con el que contaban, pues la definición de perpendicularidad requiere tener ángulos rectos, no de medida $90^{\circ}$. En [358] y [360] establece que los ángulos miden $90^{\circ}$ (datos); en [362] seńala lo escrito en la columna Qué uso la definición de rectas perpendiculares (garantía), y de acuerdo con las intervenciones [368-384] establece la perpendicularidad de los segmentos (conclusión). Aunque menciona que lo anterior también se cumple para mostrar que $\overline{I J} \perp \overline{Y A}$ no lo reporta en el esquema-deducción.

A continuación, Cristian y Diana escriben el tercer argumento.

394. Cristian: La definición de ángulos congruentes. Cópiela que esa es la que vamos a hacer.

396. Cristian: Aaaah. Pues lo mismo que acá [señala la columna Qué sé del segundo argumento: $\angle I K C \quad 90^{\circ}, \angle I K Y \quad 90^{\circ}$ (figura 9) $]$.

397. Diana: Que son ángulos rectos. Algo así había dicho. $[\ldots]$

405. Diana: Ya, ya, ya [escribe $\angle C K I$ y $\angle Y K I$ son ángulos rectos]. ¿¿ntonces? ¿Qué concluiríamos? Que el ángulo $C, K, I$ es congruente con $Y, K, I$ [escribe $\angle C K I \cong \angle Y K I]$. Tenemos tres hipótesis [argumentos].

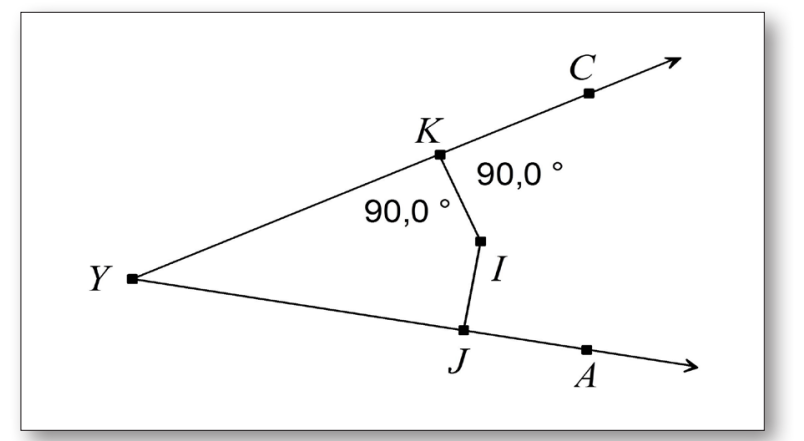

Fig. 9. Representación de la situación en Cabri.

Para este tercer argumento, Cristian desarrolla un argumento deductivo completo que Diana transforma, pues escribe que $\angle C K I$ y $\angle Y K I$ son rectos (datos). La garantía la provee Crsitian cuando le solicita a Diana que copie la definición de ángulos congruentes [394], y en [405] Diana escribe la conclusión: $\angle C K I \cong \angle Y K I$.

Cuando Diana menciona que hay que elegir una de las tres hipótesis [405], se evidencia que es consciente de que aún no tienen una demostración y que deben organizar los tres argumentos escritos en la hoja (logro) (figura 10).

\begin{tabular}{|c|c|c|}
\hline Qué sé & Qué uso & Qué concluyo \\
\hline $\begin{array}{l}\overline{K I} \text { tiene la misma } \\
\text { medida con } \overline{I J}\end{array}$ & $\begin{array}{l}\text { D. Segmentos } \\
\text { congruentes }\end{array}$ & $\overline{K l} \cong \overline{I J}$ \\
\hline $\begin{array}{l}\angle I K C 90^{\circ} \\
\angle I K Y 90^{\circ}\end{array}$ & $\begin{array}{c}\text { D. Rectas } \\
\text { perpendiculares }\end{array}$ & $\overline{I K} \perp \overline{Y C}$ \\
\hline $\begin{array}{l}\angle C K I \text { y } \angle Y K I \quad \text { son } \\
\text { ángulos rectos }\end{array}$ & $\begin{array}{l}\text { D. Ángulos } \\
\text { congruentes }\end{array}$ & $\angle C K I \cong \angle Y K I$ \\
\hline
\end{tabular}

Fig. 10. Transcripción de los AS escritos por los estudiantes. 
A continuación, ellos hacen referencia a los tres argumentos completos que diligenciaron en el esquema-deducción y que no están encadenados para poder justificar la afirmación.

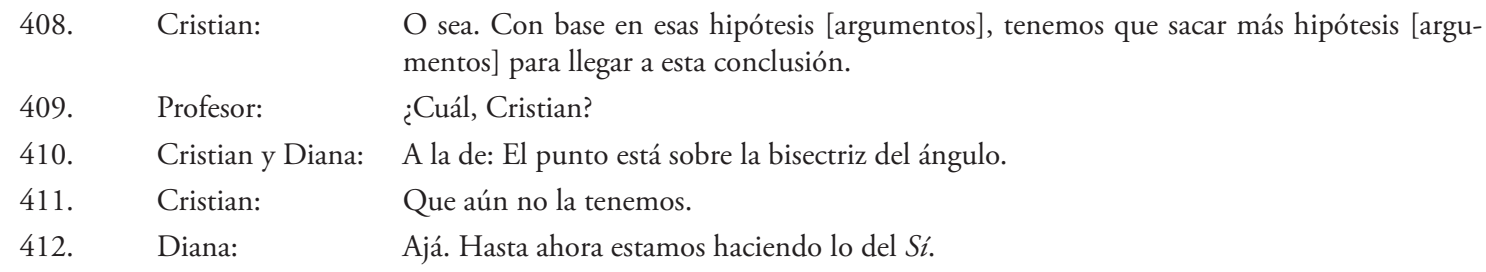

Cristian sabe que para justificar la afirmación se requieren más argumentos aparte de los tres ya formulados [408]. Ambos estudiantes reconocen que el antecedente [412] contiene información con la cual deben empezar la justificación (logro).

\section{Episodio 3: Elaboran el segundo y tercer AP}

Para continuar con la construcción de la justificación, Cristian menciona de nuevo que basándose en lo que se sabe (antecedente de la condicional) se debe justificar lo que plantea la afirmación (consecuente de la condicional). Diana propone que el primer argumento (AS) completo escrito en el esquema sea el primer paso de la justificación (AP), pero ante la solicitud de Cristian no sabe cómo justificar su propuesta. Frente a esta situación, el profesor interviene para indicar que la propuesta de Diana de comenzar con el primer argumento que ellos establecieron (la congruencia de los segmentos correspondientes) es correcta, pero que deben explicar por qué lo es. Por ello, Diana responde: «Pues yo sé por lo que [el punto] debía tener la misma distancia a cada lado del ángulo». Escribe nuevamente este primer argumento en el esquema-deducción como primer paso de la justificación.

En el diálogo que sigue, los estudiantes escriben la conclusión del segundo paso de la justificación cuyo dato es "IK distancia del punto a la recta» y cuya garantía es la definición de distancia de un punto a una recta, y proponen el tercer paso de esta.

814. Diana: I, Kes perpendicular con $Y, C$ [ escribe $\overline{I K} \perp \overrightarrow{Y C}$ en la columna Qué concluyo del segundo paso]. [...] Ahora escribimos eso $[\overline{I K} \perp \overrightarrow{Y C}$ ] acá [en la columna Qué sé del tercer paso].

$[\ldots]$

817. Cristian: Se hace lo mismo con el de abajo [con el $\overrightarrow{Y A}$ para plantear que $\overline{I J} \perp \overrightarrow{Y A}$ ].

$[\ldots]$

822. Diana: Sí [como tercer paso, escribe en la columna Qué sé: IJ distancia del punto a la recta; Qué uso: Definición distancia de un punto a una recta; Qué concluyo: $\overline{I J} \perp \overrightarrow{Y A}$ ]. Ya. Ahora, eso sí lo escribimos aquí abajo [en la columna Qué sé del cuarto paso], esas conclusiones.

Cristian propone incluir como dato del tercer paso no solamente lo que Diana menciona $(\overrightarrow{I K} \perp \overrightarrow{Y C})$ sino también $\overline{I J} \perp \overrightarrow{Y A}$. En [822] Diana escribe su argumento en el esquema-deducción. De esta forma, queda establecido el tercer paso de la justificación. Tanto en esta intervención como en la [814] se evidencia que Diana comprende que una justificación implica el encadenamiento de argumentos porque menciona que la conclusión que acaban de obtener pasa a ser el dato de otro paso (logro). 


\section{Episodio 4: Construyen el cuarto y quinto AP}

En lo que sigue, Cristian y Diana empiezan a elaborar el cuarto paso de la justificación.

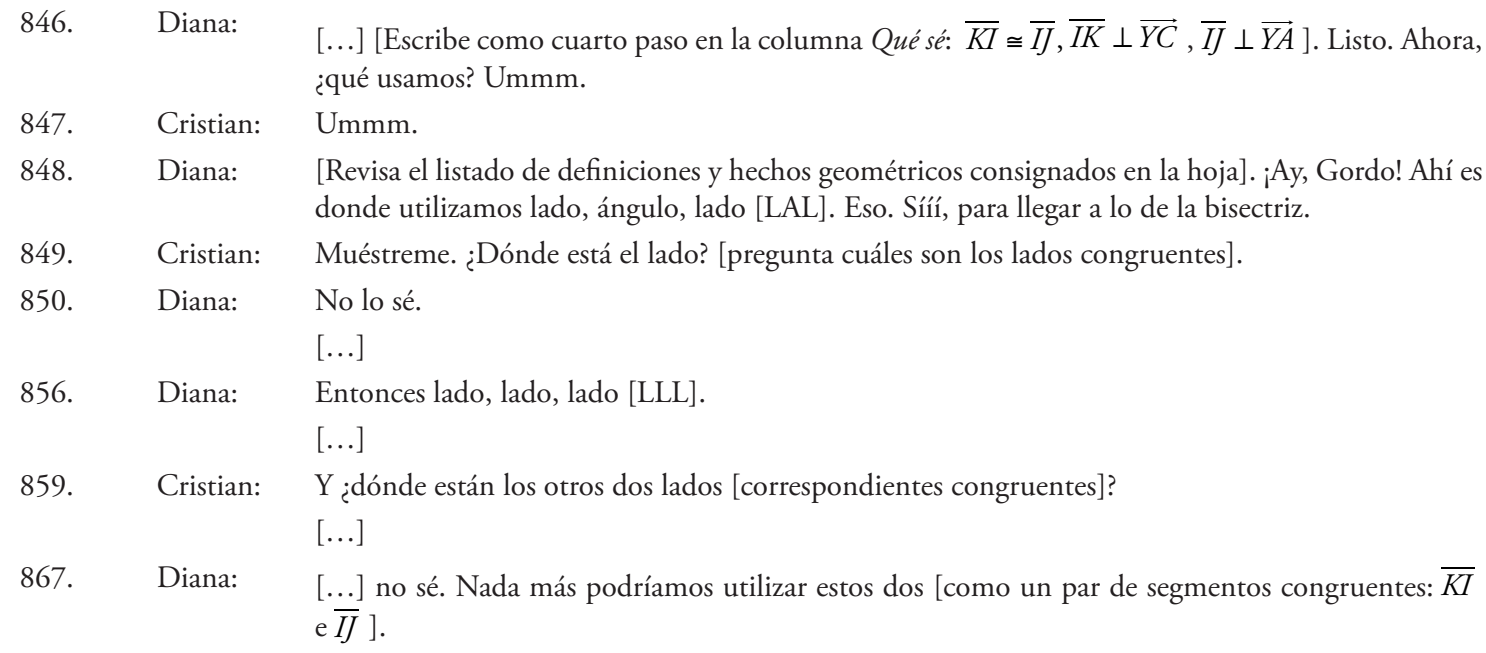

$\mathrm{Al}$ inicio de este fragmento, Diana presenta un argumento deductivo completo [846, 848] en el cual los nuevos datos son las conclusiones de los tres pasos anteriores, la garantía es el hecho geométrico LAL para la congruencia de triángulos y la conclusión es que el punto está en la bisectriz del ángulo. Demuestra su incertidumbre (cualificador modal) respecto a su argumento al decir «No lo sé», que expresa debido a la refutación a la garantía que hace Cristian [849]. Por esto, ella propone otra garantía [856] y expresa la misma inseguridad cuando Cristian pregunta en [859] por la congruencia del otro par de lados. Lo que se destaca de este fragmento son dos aspectos: primero, Diana reconoce que el camino para llegar a la conclusión de la afirmación no es inmediato y que necesitan de la congruencia de triángulos para establecerlo, pese a que en la figura no se visualizan triángulos (logro); segundo, Cristian cuestiona si se tienen los datos que permiten el uso de las garantías propuestas por Diana (logro).

Después, el profesor les pregunta qué garantía podrían usar teniendo en cuenta los datos que han establecido. A partir de ello, los estudiantes completan el cuarto paso de la justificación.

871. Diana: Usamos la definición de rectas perpendiculares. Entonces esto $[\overline{K I} \cong \overline{I J}]$ todavía no lo usaríamos. Ah, estas dos [señala con el lápiz $\overline{I K} \perp \overrightarrow{Y C}$ y $\overline{I J} \perp \overrightarrow{Y A}$ ] y esta todavía [seńala y borra en la columna Qué sé del cuarto paso: $\overline{K I} \cong \overline{I J}$. Y en la columna Qué uso escribe: «Definición de rectas perpendiculares»].

872. Cristian: Ahí ya sabemos que son ángulos de $90^{\circ}$.

$[\ldots]$

875. Diana: [Lee la definición de rectas perpendiculares]. Dos rectas son perpendiculares si se intersecan y determinan cuatro ángulos rectos...

876. Cristian: Sabemos que, ahora sí sabemos que son ángulos rectos.

877. Diana: Pues sí pero ¿̇cómo escribo en lo Qué concluyo?

878. Cristian: Pues mire la gráfica. Si esto es perpendicular y esto es perpendicular [marca los ángulos $\angle I K C, \angle I K Y, \angle I I Y, \angle I I A$ para indicar que son ángulos rectos].

$[\ldots]$

922. Diana: [...] Ahora sí. Ángulo $I, K, C \ldots$ [escribe en la columna Qué concluyo del cuarto paso: " $\angle I K C, \angle I K Y, \angle I J Y, \angle I J A$ son ángulos rectos»]. 
Diana se centra en la perpendicularidad porque reconoce que le puede dar información sobre ángulos. Cristian propone como conclusión que la medida de los ángulos es $90^{\circ}$, pero es Diana quien establece que los ángulos son rectos a partir de la definición de rectas perpendiculares (garantía). Con ayuda de la gráfica, Cristian le indica a Diana cuáles son los ángulos rectos involucrados (conclusión). De este modo, la gráfica se convierte en fuente de información con la que se puede inferir lo que tendría que ser verdadero.

Diana y Cristian escriben el quinto paso con la ayuda del profesor. Como garantía usan el hecho geométrico que Cristian mencionó (congruencia de ángulos rectos). Escriben en la columna Qué concluyo: $\angle I K C \cong \angle I K Y, \angle I J Y \cong \angle I J A, \angle I K C \cong \angle I J Y, \angle I K Y \cong \angle I J A$, y en la columna Qué sé: " $\angle I K C$, $\angle I K Y, \angle I J A$ son ángulos rectos», datos que Diana propone usando la conclusión del paso anterior.

\section{Conflictos epistémicos en torno a la bisectriz}

En este apartado, describiremos dos conflictos epistémicos que surgieron alrededor del concepto bisectriz. El primero de ellos lo denominamos conflicto epistémico de representación, que se evidencia en las siguientes intervenciones de Cristian. Consistió en rechazar la representación de un rayo en el interior del ángulo, pues creía que dibujarlo significaba atribuirle la propiedad de ser bisectriz.

103. Cristian: ¿Qué es lo que tenemos que concluir? El punto está sobre la bisectriz. O sea que aún no sabemos la bisectriz. O sea, no hemos sacado esto [borra la bisectriz del $\angle C Y A$ ]. Aún no hemos sacado esto. $[\ldots]$

$[\ldots]$

472. Diana: Yo veo dos triángulos congruentes: $J, Y, I$ e $I, K, Y$.

473. Cristian: No porque... es que Diana se está confundiendo con esta línea $[\overrightarrow{Y I}$ bisectriz del $\angle C Y A]$.

474. Profesor: Déjenla. Bueno, no sé.

475. Cristian: No, porque esta línea aún no existe [indica con el cursor la bisectriz del ángulo].

Este conflicto, que requirió la intervención del profesor para sobreponerlo, se convirtió en obstáculo para desarrollar la justificación como Diana quería, usando la congruencia de triángulos. Una vez aclarado que dibujar el rayo no implicaba asegurar la propiedad de ser bisectriz, pudieron proceder con el plan de Diana y terminar la justificación.

Siguiendo la idea de Diana de demostrar la congruencia de los triángulos $I K Y$ e $I J Y$, después de aceptar que estos existen, logran establecer que el criterio adecuado es Hipotenusa-Cateto (HC), no sin antes resolver el dilema de usar la propiedad reflexiva para establecer la congruencia del $\overline{Y I}$ consigo mismo. El comportamiento de los estudiantes durante la construcción de los pasos sexto a noveno de la demostración fue muy similar a lo que hemos descrito, razón por la cual presentamos el análisis de manera sucinta (figura 11).

Para el sexto paso, Cristian formula un argumento deductivo completo en el cual el dato es el $\overline{Y I}$ que señala en la figura, la conclusión es que el $\overline{Y I}$ es congruente consigo mismo y la garantía que provee es la propiedad que él llama «inversa». Diana indica que la propiedad a la que él se refiere se llama «reflexiva». Con la ayuda del profesor, establecen el séptimo paso de la demostración. Él interviene para recordarles que el hecho geométrico HC exige establecer antes que los triángulos sean rectángulos, cosa que hacen a partir de la conclusión del paso 4. Luego Cristian establece la congruencia de los triángulos $\triangle I K Y$ e $\Delta I J Y$ (octavo paso) con un argumento deductivo incompleto, pues no menciona como datos que los triángulos son rectángulos. Al consignar este paso en la tabla, incluyen como dato lo que no mencionó Cristian pero no todos los datos requeridos para concluir la congruencia 
$(\overline{I J} \cong \overline{I K}, \angle I J Y \cong \angle I K Y$ e $\overline{I Y} \cong \overline{I Y})$. Para el noveno paso, surgió un desacuerdo entre Diana y Cristian en torno a la garantía para concluir la congruencia de los ángulos $\angle I Y K$ e $\angle I Y J$. Diana propone la definición de ángulos congruentes y Cristian el hecho de ser ángulos de triángulos congruentes. El argumento de cada uno es deductivo completo, el primero incorrecto y el segundo correcto.

\begin{tabular}{|c|c|c|c|}
\hline Paso & Qué sé & Qué uso & Qué concluyo \\
\hline Sexto & $\begin{array}{l}\text { Que el } \Delta K I Y \text { y el } I J Y \\
\text { comparten el } \overline{Y I}\end{array}$ & Propiedad Reflexiva & $\overline{Y I} \cong \overline{Y I}$ \\
\hline Séptimo & $\begin{array}{l}\angle Y J I, \quad \angle Y K I \quad \text { son } \\
\text { ángulos rectos }\end{array}$ & $\begin{array}{l}\text { D. Triángulo } \\
\text { Rectángulo }\end{array}$ & $\begin{array}{c}\Delta I K Y \text { y } \triangle I J Y \text { son } \\
\text { triángulos rectángulos }\end{array}$ \\
\hline Octavo & $\begin{array}{l}\triangle I K Y \text { y } \triangle I J Y \text { son } \\
\text { triángulos rectángulos }\end{array}$ & $\begin{array}{l}\text { HG. Hipotenusa- } \\
\text { Cateto }\end{array}$ & $\Delta I K Y \cong \Delta I J Y$ \\
\hline Noveno & $\Delta I K Y \cong \Delta I J Y$ & $\begin{array}{l}\text { D. Triángulos } \\
\text { congruentes }\end{array}$ & $\angle K Y I \cong \triangle J Y I$ \\
\hline
\end{tabular}

Fig. 11. Transcripción de los pasos sexto a noveno de la demostración escritos por los estudiantes.

En el siguiente diálogo, los estudiantes comienzan a escribir el décimo paso de la justificación.

\begin{tabular}{|c|c|c|}
\hline 1389. & Cristian: & Ahora sabemos esto [señala la conclusión del noveno paso: $\angle K Y I \cong \angle J Y I$ ]. \\
\hline 1390. & Diana: & [Escribe en la columna Qué sé del décimo paso: $\angle K Y I \cong \angle J Y I]$. \\
\hline 1391. & Cristian: & Y ahí podemos utilizar la [definición] de bisectriz de un ángulo. \\
\hline 1392. & Diana: & $\begin{array}{l}\text { [Escribe en la columna Qué uso del décimo paso: Definición de bisectriz de un ángulo]. Y si } \\
\text { utilizamos la [definición] de bisectriz de un ángulo concluimos que... concluimos... }\end{array}$ \\
\hline 1393. & Cristian: & ¿A qué tenemos que llegar? \\
\hline 1394. & Diana: & $\begin{array}{l}\text { Pues dice que el punto está sobre la bisectriz del ángulo [señala el consecuente de la afirma- } \\
\text { ción]. Entonces concluimos que... }\end{array}$ \\
\hline 1395. & Cristian: & Ya acabamos, ¿no? \\
\hline 1396. & Diana: & Pero debemos concluir algo. \\
\hline 1397. & Cristian: & Pues eso. \\
\hline 1398. & Diana: & ¿Qué? \\
\hline 1399. & Cristian: & El entonces. \\
\hline $1400-1413$. & & [El profesor interviene para que los estudiantes puedan escribir la respectiva conclusión.] \\
\hline 1414. & Cristian: & $\begin{array}{l}\text { Pues conseguimos una bisectriz. Si son congruentes }[\angle K Y I \text { y } \angle J Y I] \text {, conseguimos una bi- } \\
\text { sectriz. Entonces conseguimos la bisectriz } Y, I \text {. }\end{array}$ \\
\hline
\end{tabular}

Cristian presenta un argumento deductivo completo en el que se tiene explícitamente: dato, $\angle K Y I \cong \angle J Y I$; garantía, la definición de bisectriz de un ángulo, y conclusión, que el punto está sobre la bisectriz del ángulo. Sin embargo, Diana no acepta [1395] la conclusión que propone Cristian porque usar dicha garantía llevaría a concluir que el $\overrightarrow{Y I}$ es la bisectriz del $\angle C Y A$, pero no que el punto $I$ pertenezca a la bisectriz. Con esta objeción, él revisa su argumento y cambia su conclusión.

Finalizan la demostración de la conjetura [1437-1505] colocando como conclusión que $\overrightarrow{Y I}$ es la bisectriz del $\angle C Y A$. El profesor se dio cuenta de que los estudiantes no quedaron conformes con dicha conclusión, pues ellos estaban acostumbrados a que se termina una demostración cuando se obtiene el consecuente de la condicional. El profesor tuvo que aclararles que ya habían acabado porque, al haber concluido que el $\overrightarrow{Y I}$ es la bisectriz, podían afirmar que el punto $I$ está en la bisectriz. Este suceso lo denominamos un conflicto epistémico de notación. Los estudiantes no comprendían que al usar el nombre para referirse al rayo $(\overrightarrow{Y I})$ se estaba indicando que el punto $I$ pertenece a dicho objeto. Tras la aclaración, ellos construyeron el último argumento que fue deductivo incompleto porque les faltó usar la definición "pertenecer a» como garantía que relaciona el dato, la bisectriz del $\angle C Y A$ es $\overrightarrow{Y I}$, con la conclusión, $I \in \overrightarrow{Y I}$. 


\section{COMENTARIOS FINALES}

Con el análisis hecho sobre las acciones de los estudiantes durante el proceso de justificación, pudimos establecer tanto los logros como los desaciertos que tuvieron. En relación con lo primero, a partir de los argumentos que señalamos en dicho proceso, identificamos la estrategia novedosa a la que recurrieron los estudiantes, que consistió en construir los AS simplemente extrayendo información de lo dado en el problema sin preocuparse por encadenarlos. Luego, reconocieron que dichos argumentos tendrían que ser algunos pasos de la demostración. Eventualmente lograron reutilizar los AS escribiéndolos como $\mathrm{AP}$ en el lugar correcto de la justificación final (figura 12). Además, nos dimos cuenta de que hubo instantes en los que Cristian y Diana se apoyaron mutuamente para construir argumentos; por eso fue necesario considerar, dentro de la tipología que empleamos, cuándo eran argumentos individuales o argumentos colectivos.

\begin{tabular}{|c|c|c|c|c|}
\hline & Qué sé & Qué uso & Qué concluyo & \multirow{4}{*}{$\begin{array}{c}\text { Argumentos situación } \\
\text { (AS) }\end{array}$} \\
\hline & \begin{tabular}{|lcl}
$\overline{K I}$ tiene la & misma \\
medida con $\bar{I}$ & \\
\end{tabular} & $\begin{array}{l}\text { D. Segmentos } \\
\text { congruentes }\end{array}$ & $\overline{K I} \cong \overline{I J}$ & \\
\hline & $\begin{array}{l}\angle I K C \quad 90^{\circ} \\
\angle I K Y 90^{\circ} \\
\end{array}$ & $\begin{array}{l}\text { D. Rectas } \\
\text { gerpendiculares }\end{array}$ & $\overline{I K} \perp \overline{Y C}$ & \\
\hline & \begin{tabular}{|lll}
$\angle C K I$ y & $\angle Y K I$ & son \\
ángulos rectos & \\
\end{tabular} & $\begin{array}{l}\text { D. Ángulos } \\
\text { congruentes }\end{array}$ & $\angle C K I \cong \angle Y K I$ & \\
\hline \multirow[t]{5}{*}{ (1) } & \begin{tabular}{|lcl}
$K I$ tiene la & misma \\
medida con $\overline{I J}$ & \\
\end{tabular} & $\begin{array}{l}\text { D. Segmentos } \\
\text { congruentes }\end{array}$ & $\overline{K I} \cong \overline{I J}$ & \multirow{5}{*}{$\begin{array}{c}\text { Argumentos paso } \\
(\mathrm{AP})\end{array}$} \\
\hline & $\begin{array}{l}\text { IK distancia del punto } \\
\text { a la recta }\end{array}$ & $\begin{array}{l}\text { D. Distancia de un } \\
\text { punto a una recta }\end{array}$ & $\overline{I K} \perp \overrightarrow{Y C}$ & \\
\hline & $\begin{array}{l}\text { IJ distancia del punto a } \\
\text { la recta } \\
\end{array}$ & $\begin{array}{l}\text { D. Distancia de un } \\
\text { punto a una recta }\end{array}$ & $\overline{I J} \perp \overrightarrow{Y A}$ & \\
\hline & $\frac{\overline{I K} \perp \overrightarrow{Y C}}{\overline{I J} \perp \overrightarrow{Y A}}$ & $\begin{array}{c}\text { D. Rectas } \\
\text { perpendiculares }\end{array}$ & $\begin{array}{ll}\angle I K C, & \angle I K Y, \quad \angle I J Y, \\
\angle I J A & \text { son ángulos } \\
\text { rectos } & \end{array}$ & \\
\hline & \begin{tabular}{|lll}
$\angle I K C$, & $\angle I K Y, \quad \angle I J Y$ \\
$\angle I J A$ & son ángulos \\
ectos &
\end{tabular} & HG. Ángulos rectos & $\begin{array}{l}\angle I K C \cong \angle I K Y \\
\angle I J Y \cong \angle I J A \\
\angle I K C \cong \angle I J Y \\
\angle I K Y \cong \angle I J A\end{array}$ & \\
\hline
\end{tabular}

Fig. 12. Transcripción de parte de la justificación presentada por los estudiantes.

También podemos afirmar que Cristian y Diana lograron comprender lo que es una demostración y aprendieron a producirla. Ambos estudiantes reconocieron el papel de cada parte de la afirmación, escrita en forma de condicional, que debían justificar, es decir, diferenciaron entre lo dado (antecedente) y la conclusión (consecuente). Por esta razón, reconocieron que debían elaborar más argumentos para llegar a la conclusión de la afirmación, luego de haber formulado los tres AS. Entendieron que para justificar la afirmación se deben encadenar argumentos, pues explícitamente indican que la conclusión de un paso pasa a ser el dato de otro. Además, reconocieron que el antecedente del elemento teórico que puede servir como garantía debe coincidir con los datos del argumento que se está construyendo.

Acerca de la construcción de una demostración, ya sea por un experto o por un novato, se tiene que no es un proceso sin altibajos. En ocasiones, como lo hicieron los estudiantes, se realizan argumentos abductivos en los cuales, conocida la conclusión que se quiere obtener, se buscan los datos y la garantía que permiten llegar a ella. Por otro lado, buscar elementos teóricos para los cuales la hipótesis coincide con los datos que se tienen a mano es un acto de un aprendiz que entiende el esquema de razonamiento válido (Modus Ponendo Ponens) que permite deducir información a partir de los datos usando el elemento teórico correspondiente, pero que no tiene dominio completo del sistema teórico con el que cuenta. Este acto puede parecer ser un ejercicio de emparejamiento pero no lo es. 
Los logros también se relacionan, en términos generales, con el uso correcto que hicieron los estudiantes de la gráfica para extraer y reportar información que se acepta como verdadera; además es logro la formulación de argumentos completos y correctos.

La caracterización de los argumentos elaborados por los estudiantes nos permitió establecer varios desaciertos cuando se está aprendiendo a demostrar. Primero, no poder traducir la definición de distancia de un punto a una recta a la situación del problema, lo cual dificultó el desarrollo inicial de la justificación. Segundo, incapacidad para generalizar situaciones que estudian de manera empírica (medida de segmentos) con el uso de geometría dinámica. Tercero, considerar que una figura debe representar fielmente los datos incluidos en el antecedente de una proposición condicional por demostrar (a pesar de que visualmente los segmentos no eran perpendiculares a los lados del ángulo, esta propiedad se tomaba como dada de acuerdo con las condiciones del problema). Cuarto, formular argumentos incompletos o argumentos completos pero incorrectos porque usan garantías que no se corresponden con los datos.

Los desaciertos también están asociados con los dos dilemas epistémicos que surgieron. El primero relacionado con la representación geométrica de figuras y la información que sí se puede deducir de ella; el segundo, con la notación y las propiedades que se establecen a través de ella sobre los puntos que se usan en esta. Por esto, destacamos que el papel del profesor quien, como experto, debe estar atento a estos conflictos e intervenir oportunamente para que los estudiantes logren superarlos comprensivamente y dejen de ser obstáculos para construir demostraciones.

\section{DISCUSIÓN}

De lo expuesto anteriormente quedan preguntas que podrían ser tema de otras investigaciones. ¿ Será que la estrategia que usaron los estudiantes de inicialmente construir argumentos AS y luego buscar si estos se pueden encadenar para producir una demostración es un procedimiento que un profesor debe proponer cuando intenta enseñar cómo justificar afirmaciones matemáticas con argumentos teóricos? ¿Se favorece la construcción de demostraciones cuando se trabaja en grupo en contraposición del trabajo individual? Los dilemas epistémicos presentados aquí habrían llevado al fracaso en la producción de la justificación, si no hubiera estado el profesor presente para reconocerlos. Por ello nos preguntamos ¿qué otro tipo de dilemas pueden estar afectando la posibilidad de construir demostraciones? ¿Cómo puede el profesor identificar los dilemas si él no puede estar atento a lo que sucede con cada estudiante en el aula? Cosas que los profesores creemos son de fácil comprensión, como la relación entre el nombre de una figura y la pertenencia de puntos a esta, ¿pueden ser asuntos problemáticos para los estudiantes?

\section{REFERENCIAS BIBLIOGRÁFICAS}

Arzarello, F.; Olivero, F; Paola, D. y Robutti, O. (2007). The transition to formal proof in geometry. En P. Boero (ed.). Theorems in school. From history, epistemology and cognition to classroom practice. Rotterdam: Sense Publishers, pp. 305-323.

Camargo, L.; Samper, C. y Perry, P. (2006). Una visión de la actividad demostrativa en geometría plana para la educación matemática con el uso de programas de geometría dinámica. Lecturas Matemáticas, 27 (especial), pp. 371-383.

Cohen, L. y Manion, L. (1990). Métodos de investigación educativa (trad. Francisco Agudo López). Madrid: Ediciones La muralla (primera edición en inglés, 1989). 
De Villiers, M. (1993). El papel y la función de la demostración en matemáticas. Epsilón, 26, pp. $15-29$.

Fonseca, J.; Lara, L. F. y SAmper, C. (2012). Un camino hacia la actividad demostrativa. En G.

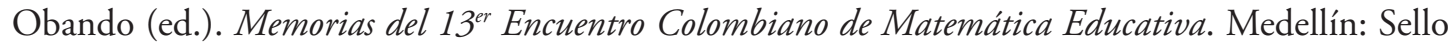
Editorial Universidad de Medellín, pp. 898-906.

Fonseca, J. y Lara, L.F. (2013). Análisis del comportamiento racional y argumental de estudiantes de grado noveno cuando trabajan en grupo dentro de un ambiente que propicia la actividad demostrativa. Tesis de maestría. Bogotá: Universidad Pedagógica Nacional.

Godino, J. y Recio, A. M. (2001). Significados institucionales de la demostración. Implicaciones para la educación matemática. Enseñanza de las Ciencias, 19(3), pp. 405-414.

Jones, K. (2000). Providing a foundation for deductive reasoning: Students' interpretations when using dynamic geometry software and their evolving mathematical explanations. Educational Studies in Mathematics, 44(1-3), pp. 55-85.

http://dx.doi.org/10.1023/A:1012706005027

http://dx.doi.org/10.1023/A:1012789201736

LAVY, I. (2004). Kinds of arguments emerging while exploring in a computerized environment. En Proceedings of the 28th Conference of the International, vol. 3, pp. 185-192.

Pedemonte, B. (2005). Quelques outils pour l'analyse cognitive du rapport entre argumentation et démonstration. Recherche en Didactique des Mathématiques, 25, pp. 313-348.

Perry, P.; Camargo, L.; Samper, C. y Rojas, C. (2006). Actividad demostrativa en la formación inicial del profesor de matemáticas. Bogotá: Fondo Editorial de la Universidad Pedagógica Nacional.

Perry, P.; Samper, C.; Camargo, L. y Molina, Ó. (2013). Innovación en un aula de geometría de nivel universitario. En C. Samper y Ó. Molina. Geometría Plana: Un espacio de aprendizaje. Bogotá: Fondo editorial de la Universidad Pedagógica Nacional, pp. 13-36

Rodríguez, F. y Gutiérrez, A. (2012). Software DeMMaTTouL: una herramienta para la investigación sobre la estructura argumentativa de la demostración. En A. Estepa, Á. Contreras, J. Deulofeu, M. C. Penalva, F. J. García y L. Ordóńez (eds.). Investigación en Educación Matemática XVI. Jaén: SEIEM, pp. 457-468.

Samper, C.; Molina, Ó. y Echeverry, A. (2011). Elementos de Geometría. Bogotá: Fondo editorial de la Universidad Pedagógica Nacional.

Selden, A. y Selden, J. ( 2011). Mathematical and non-mathematical university students' proving difficulties. En L. R. Wiest y T. D. Lamburg (eds.). Proceedings of the 33rd Annual Conference of the North American Chapter of the International Group for the Psychology of Mathematics Education. Reno, NV: University of Nevada, Reno, pp. 675-683.

Toulmin, S. E. (2003). The uses of argument. Cambridge University Press. 


\title{
Successes and failures when learning to prove
}

\author{
Luis Fernando Lara Quintero \\ Secretaría de Educación Distrital, Bogotá, Colombia \\ luisfernandolara26@yahoo.es \\ Carmen Samper \\ Universidad Pedagógica Nacional, Departamento de Matemáticas, Bogotá, Colombia \\ csamper@pedagogica.edu.co
}

With the intention of introducing ninth grade students into the practice of mathematical argumentation, we modified the usual teaching methodology by introducing the use of dynamical geometry software in class. This led the students to carry out proving activity (Perry, Samper, Camargo \& Molina, 2013), as they explored a geometric situation with dynamic geometry, formulated a conjecture about the geometric fact - previously unknown to them, that underlies the situation, and tried to prove the conjecture. This activity fostered argumentation and promoted student participation. From the analysis of the students' arguments using Toulmin's model (Rodríguez \& Gutiérrez, 2012), it was possible to identify student successes and failures with respect to what proving is and how to produce a proof. We hope to contribute somehow to research results about the difficulties students have when learning to prove (Selden \& Selden, 2011; Perry, Camargo, Samper \& Rojas, 2006).

According to Perry et. al. (2013), proving activity consists of two processes. The first one, conjecturing, consists of all the actions that lead to the establishment of a conjecture based on evidence provided by the exploration of a situation using dynamic geometry. The second one, justifying, refers to actions to validate the conjecture within a theoretical system or with empirical explanations, according to the school level. Toulmin's basic model of an argument consists of three principal elements: data or information, conclusion expressed as a statement that is believed to be a consequence of the data, and warrant, a rule (axiom, definition or theorem) that relates the data with the conclusion.

In our research, we adopted a qualitative methodology centered on a descriptive-interpretative trend that corresponds to a case study (Cohen \& Manion, 1990). The type of case study was non-participant because the teacher avoided involvement in the students' argumentation, and structured because a specific methodological approach to teaching was used. The approach consists of three elements. The tasks that favor exploration and formulation of conjectures; the social interaction between students and with the teacher, which enables communicating and discussing ideas that are accepted or rejected through argumentation; the use of dynamic geometry that favors geometric constructions, and exploring geometric properties to produce conjectures.

We designed and implemented a teaching sequence in a public school in Bogotá (Colombia) with ninth graders (14-16 years old) during two months. The case study was over the activity that two students carried out when solving two problems at the end of the two months. The geometric fact they had to establish as a conjecture and then prove was that the locus of points that are equidistant from the sides of an angle is the angle bisector.

Various are the successes we identified with our analysis of the arguments. These are: the students' novel strategy of initially constructing three arguments by just extracting information from the data given in the problems without trying to link them together; recognizing these should become steps of the required proof and being able to incorporate them when they were producing the proof; recognizing the role of the antecedent and consequent of the statement they were trying to prove; realizing that the antecedent of the theoretical element they wanted to use as a warrant in an argument must coincide with the data they have on hand; correctly using a graphic representation to extract or record valid information; and formulating some complete and correct arguments.

Some of the failures were: not being able to translate the distance from a point to a line definition in terms of the specific problem they were solving; difficulty in generalizing information they obtained empirically when using dynamic geometry; considering that a drawn figure must faithfully represent the given properties; and formulating incomplete or incorrect arguments. Related to these are the two epistemic dilemmas that surfaced. The first one had to do with the geometric representation of the figure they were using to visualize the situation. One of the students would not permit drawing a ray with endpoint the angle vertex $Y$ and containing a point $I$ equidistant to the sides of the angle because they had not yet proven that it was the bisector. The second one is related to geometric notation and the properties established when using it. When the students finally proved that ray $Y I$ was the bisector of $\angle K Y J$, they didn't understand that this meant point $I$ belongs to the angle bisector, which is what they had to prove. This brings forth the importance of the teacher's role. He must pay attention to these conflicts and intervene opportunely so that the students can overcome them comprehensively and they cease being an obstacle to produce proofs. 